\title{
Adiponectin treatment attenuates inflammatory response during early sepsis in obese mice
}

\section{XianFeng Wang \\ Nancy L Buechler \\ Barbara K Yoza \\ Charles E McCall \\ Vidula Vachharajani}

Department of Anesthesiology, Medicine and Surgery, Wake Forest School of Medicine, Winston-Salem, NC, USA
Correspondence: Vidula Vachharajani Department of Anesthesiology and Medicine, Wake Forest School of Medicine, Medical Center Blvd, Winston-Salem, NC 27I57, USA

$\mathrm{Tel}+\mathrm{I} 3367164498$

Fax +I 3367168190

Emailvachhar@wakehealth.edu
This article was published in the following Dove Press journal:

Journal of Inflammation Research

5 October 2016

Number of times this article has been viewed

Background: Morbid obesity increases the cost of care in critically ill patients. Sepsis is the leading cause of death in noncoronary intensive care units. Circulating cell-endothelial cell interactions in microcirculation are the rate-determining factors in any inflammation; obesity increases these interactions further. Adiponectin deficiency is implicated in increased cardiovascular risk in obese patients. We have shown that adiponectin deficiency increases microvascular dysfunction in early sepsis. In the present study, we investigated the effect of adiponectin replacement on nutritionally obese mice with early sepsis.

Methods: We used cecal ligation and puncture model of sepsis in mice with diet-induced obesity (DIO) vs control diet (CTRL), with or without adiponectin treatment. We studied leukocyte/ platelet adhesion in the cerebral microcirculation in early sepsis. We also studied the effect of adiponectin on free fatty acid (FFA)-fed and lipopolysaccharide-stimulated bone marrow-derived macrophages (BMDM) for mechanistic studies.

Results: Leukocyte and platelet adhesion increased in the cerebral microcirculation of DIO and CTRL mice with early sepsis vs. sham; moreover cell adhesion in DIO-sepsis group was significantly higher than in the CTRL-sepsis group. Adiponectin replacement decreased leukocyte/platelet adhesion in CTRL and DIO mice. In FFA-fed BMDM, adiponectin treatment decreased tumor necrosis factor-alpha mRNA expression and increased sirtuin-1 (SIRT1) mRNA expression. Furthermore, using BMDM from SIRT1 knockout mice, we showed that the adiponectin treatment decreased inflammatory response in FFA-fed BMDM via SIRT1-dependent and -independent pathways.

Conclusion: Adiponectin replacement attenuates microvascular inflammation in DIO-sepsis mice. Mechanistically, adiponectin treatment in FFA-fed mouse macrophages attenuates inflammatory response via SIRT1-dependent and -independent pathways.

Keywords: sepsis, obesity, adiponectin, leukocyte adhesion, inflammation, sirtuin-1

\section{Introduction}

Sepsis, the leading cause of death in noncoronary intensive care units, kills over 200,000 patients per year with annual cost of care over US\$16 billion in the US alone. ${ }^{1,2}$ Microvasculature with its strategic place between the systemic circulation and local tissue environment plays a crucial role in the inflammatory response. ${ }^{3}$ Evidence suggests that circulating cell-endothelial cell interactions in microcirculation are the ratedetermining factors in any inflammatory response, including sepsis. ${ }^{4}$ We have shown previously that obesity induces significantly increased circulating cell-endothelial cell interactions during early sepsis. We have also shown that this increase in microvascular inflammation is via modulation of the adhesion molecule/PSGL-1 expression. ${ }^{5,6}$ 
Obesity endemic affects one in three individuals in the US with the cost of health care expected to increase by over US $\$ 500$ billion by the year 2030 if the trend continues. ${ }^{7}$ Although increased mortality in obese, septic patients is controversial, evidence suggests that obesity increases the risk of sepsis. ${ }^{8}$ In addition, obesity is known to further increase morbidity and resource utilization in already expensive care of critically ill patients. ${ }^{9,10}$ Adipose tissue is not merely a fat storage organ, but rather a "motor" for inflammatory response. ${ }^{11}$ Adipose tissue secretes various pro- and antiinflammatory adipokines that are extensively studied in various inflammatory conditions so far. ${ }^{12-15}$ Of particular importance is adiponectin. Adiponectin is secreted by differentiating adipocytes and acts as an "anti-inflammatory" molecule. Its deficiency is implicated in increased cardiovascular risk in patients with metabolic syndrome/obesity. ${ }^{16,17}$ Evidence suggests that adiponectin sensitivity is decreased in lean and obese individuals with sepsis. ${ }^{18} \mathrm{We}$ and others have shown previously that adiponectin deficiency is associated with exaggeration of microvascular inflammation in early sepsis. ${ }^{19}$ However, the effect of adiponectin treatment on early sepsis associated with obesity is not known to our knowledge.

We have studied early sepsis using leptin-deficient ob/ob and leptin-resistant $\mathrm{db} / \mathrm{db}$ and melanocortin receptor 4 knockout (MC4R KO) mice. In all these models of obesity, we have shown that there is exaggeration of inflammatory response in early sepsis. ${ }^{5,20} \mathrm{We}$ focused mainly on the endothelial dysfunction during those studies and showed that obesity accentuates microvascular inflammation via upregulation of adhesion molecule expression in various microcirculatory beds. Using adiponectin deficiency as a surrogate marker for "obesity phenotype" in early sepsis, we also showed that the increased microvascular inflammation occurs via modulation of adhesion molecule expression. ${ }^{19}$ Thus, while we have previously studied the effect of adiponectin deficiency on the endothelial dysfunction, the effects of adiponectin treatment on obese mice with sepsis and on immune cell function are not well characterized. In the present study, we investigated the effect of adiponectin treatment on nutritionally obese (diet-induced obesity[DIO]) mice and free fatty acid (FFA)-fed bone marrow-derived macrophages (BMDM) with lipopolysaccharide (LPS) stimulation to elucidate the effect of adiponectin treatment on microvascular inflammation in vivo and fat-fed macrophages in vitro.

\section{Materials and methods Animals}

The study was approved by the Institutional Animal Care and Use Committee of the Wake Forest School of Medicine, and the experiments were performed according to the National Institute of Health (NIH) guidelines for animal research. The DIO mice and their corresponding control (CTRL) were purchased from Jackson Laboratories (Bar Harbor, ME, USA). These mice were developed by feeding them with diets from Research Diets Inc., starting at the age of 6 weeks. The DIO mice were fed with diet containing 60\% fat (cat no: D12492; Research Diets Inc.), while the CTRL mice were fed with control diet containing 10\% fat (cat no: D12450B; Research Diets Inc.). These mice were 13-15 weeks of age at the time of conductance of experiments. Six-eight-week-old C57B1/6 (wild type [WT]) mice were purchased from Jackson Laboratories for isolation of BMDM. Myeloid SIRT1 knockout (SIRT1KO) mice $^{21}$ were generous gift from Dr Hang Shi (Georgia State University). These mice were created by crossing SIRT1 flox/ flox (fl/fl) mice, in which the SIRT1 allele contains a loxpflanked exon 4, with lysozyme Cre (lys-Cre) mice, in which Cre is specifically expressed in myeloid lineage cells including macrophages and monocytes. All animals were housed with a 12-hour light/dark cycle in a temperature-controlled facility and had free access to water and food.

\section{Cecal ligation and perforation}

All mice were pretreated with two doses of recombinant murine adiponectin (gAcrp30) (PeproTech, Rocky Hill, NJ, USA) $25 \mu \mathrm{g} /$ dose or vehicle (normal saline) as indicated, 18.5 hours prior to cecal ligation and puncture. Mice were anesthetized_with intramuscular (IM) ketamine hydrochloride $(150 \mathrm{mg} / \mathrm{kg})$ and xylazine $(7.5 \mathrm{mg} / \mathrm{kg})$. Cecal ligation and perforation (CLP) was performed as previously described. ${ }^{5,20}$ Briefly, a $2 \mathrm{~cm}$ midline incision in the anterior abdominal wall was made; the cecum was isolated, tightly ligated (precaution taken to not cause intestinal obstruction), and perforated three times (upper, middle, and lower third) using a 20-gauge needle. Subsequently, a small amount of fecal content was squeezed and spread around the cecum and the incision was closed in two layers followed by $1 \mathrm{~mL}$ of normal saline subcutaneously (fluid resuscitation). Sham mice underwent laparotomy similar to CLP but without ligation and puncture.

\section{Intravital videomicroscopy of cerebral microcirculation}

The cerebral microcirculation of DIO and CTRL mice was observed by intravital microscopy at 4 hours following CLP/ sham surgery by craniotomy as described previously. ${ }^{5,20}$ Briefly, mice were anesthetized with IM ketamine hydrochloride $(150 \mathrm{mg} / \mathrm{kg})$ and xylazine $(7.5 \mathrm{mg} / \mathrm{kg})$. The left femoral artery was cannulated for monitoring mean arterial 
blood pressure (MAP), and the femoral vein was cannulated for intravenous administration of platelets and/or acridine orange. Precautions were taken to maintain the core body temperature $\left(35^{\circ} \mathrm{C} \pm 0.5^{\circ} \mathrm{C}\right)$. Subsequently, a tracheotomy was performed, the head of each mouse was fixed in a stereotaxic frame, left craniotomy was performed (dura was carefully left intact), a drop of normal saline was placed on the window, and a cover glass was placed on the cranial window. The animals were allowed to stabilize for 30 minutes on a mechanical ventilator prior to observation of 3-5 cerebral postcapillary venules/mouse (just prior to penetration into the cerebral cortex) under an upright Nikon microscope.

\section{Circulating cell-endothelial cell interactions}

Leukocytes were labeled in vivo by injecting acridine orange $(0.05 \%$ in $100 \mu \mathrm{L})$ intravenously and recorded before infusion of platelets labeled ex vivo with carboxyfluorescein diacetate succinimidyl ester $(90 \mu \mathrm{M})$ to allow separate monitoring of both cell types in each venule, as platelets were observed after significant acridine orange-related fluorescence was no longer detected. The details of the platelet isolation technique are as outlined previously. ${ }^{5,20}$ Platelets were isolated from matched donors, and 100 million platelets were injected per mouse. The platelet isolation procedures had no significant effect on the activity or viability of isolated platelets. ${ }^{22}$ Three to five postcapillary venules for each leukocyte and platelet adhesion were recorded via a DVD recorder and 1 minute of recording per venule was analyzed subsequently. A cell was considered adherent if it remained stationary for 30 seconds during the 1-minute recording. Three to five postcapillary venules in each mouse were assessed to quantify leukocyte and platelet adhesion. The mean of cell adhesion of each mouse was used to generate the group mean.

\section{Isolation of BMDM}

BMDM cells were isolated and cultured as described previously. ${ }^{23}$ Briefly, the femur and tibia were separated after the mouse was sacrificed. The bone marrow was flushed with phosphate-buffered saline (PBS) and cultured in sterile Petri dishes in Dulbecco's Modified Eagle's Medium (DMEM) containing $10 \%$ fetal bovine serum and $50 \mathrm{ng} / \mathrm{mL}$ of macrophage-colony stimulating factor (M-CSF) for 7 days. BMDM cells were detached with ethylenediaminetetraacetic acid/PBS and then seeded in a 24-well plate format in M-CSF containing medium.

BMDM cells were pretreated with bovine serum albu$\min (\mathrm{BSA})$ or stearic acid (FFA) $(200 \mu \mathrm{M})$ overnight in the presence or absence of adiponectin (cat: 450-21; PeproTech) to obtain a final concentration $1 \mu \mathrm{g} / \mathrm{mL}$ and then stimulated with Escherichia coli LPS (100 ng/mL) for 3 hours. Total RNA was extracted and subjected to real-time polymerase chain reaction (RT-PCR) to detect tumor necrosis factor alpha (TNF- $\alpha$ ) and SIRT1 mRNA levels.

\section{RNA extraction and RT-PCR}

RNA was extracted using TRI reagent (Molecular Research Center, Inc., Cincinnati, OH, USA). The mRNA expression levels of target genes were quantified by quantitative RT-PCR using a SensiFAST Probe Lo-ROX One-Step Kit (BIO-78005; Bioline, London, UK) as described before. ${ }^{6}$ All results were normalized to GAPDH RNA levels. Relative quantification was calculated using the DD comparative threshold formula. All samples were run in quadruplicates to calculate the average and standard error value. TaqMan primer/probes for GAPDH, TNF $\alpha$, and SIRT-1 were purchased from Thermo Fisher Scientific (Waltham, MA, USA).

\section{Statistical analysis}

All data were analyzed using GraphPad Prism 6.0 (GraphPad Software, Inc., La Jolla, CA, USA). The mean values of two populations were analyzed using an unpaired, two-tailed Student's $t$-test or Mann-Whitney test for nonparametric data sets; more than three samples were analyzed using one-way analysis of variance (ANOVA) or two-way ANOVA with Tukey's post hoc comparisons as appropriate. $P<0.05$ was designated as significant. Leukocyte and platelet adhesion in different groups was analyzed using Tukey-Kramer post hoc test (StatView; SAS Institute Inc., Cary, NC, USA). $P<0.05$ was designated as significant.

\section{Results}

\section{Body weight and MAP in CTRL and DIO sham, sepsis, and sepsis + adiponectin groups}

Table 1 shows body weight and MAP of mice from different groups. The body weights of mice in the DIO groups were significantly higher than those in the respective CTRL groups. There were no significant differences between the groups (sham vs sepsis vs sepsis + adiponectin) within the strain. While there were no significant differences in MAP in DIO vs CTRL sham mice, both DIO- and CTRL-sepsis groups had significantly lower MAP compared to the respective sham groups. MAP in the DIO-Sepsis group was significantly lower than in the CTRL-Sepsis group. Interestingly, MAP in the sepsis + adiponectin group was significantly higher than in the sepsis alone group in both CTRL and DIO mice. 
Table I Weight in grams and mean arterial blood pressure (MAP) in different groups

\begin{tabular}{lllll}
\hline & \multicolumn{2}{l}{ Body weight $(\mathrm{g})$} & \multicolumn{2}{l}{ MAP (mmHg) } \\
& Mean \pm SEM & \multicolumn{2}{l}{ Mean \pm SEM } \\
\hline \multirow{3}{*}{ Sham } & CTRL & DIO & CTRL & DIO \\
Sepsis & $26.75 \pm 0.36$ & $36.83 \pm 1.07^{\dagger}$ & $61 \pm 2.24$ & $65 \pm 4.46$ \\
Sepsis + adiponectin & $27.5 \pm 0.2$ & $38.7 \pm 2.7^{\dagger}$ & $64.2 \pm 4.6^{\#}$ & $60.7 \pm 0.5^{\#}$ \\
\hline
\end{tabular}

Notes: Body weight of CTRL and DIO sham, sepsis, and sepsis + adiponectin groups shows no significant differences from each other within the groups, and body weights of DIO mice were significantly higher than their CTRL counterparts. The MAPs in sepsis groups were significantly lower than their respective sham counterparts. The MAPs in sepsis + adiponectin groups were significantly higher compared to sepsis alone in both CTRL and DIO mice. Finally, MAP in DIOsepsis groups was significantly lower compared to $C T R L$-sepsis groups. ${ }^{*} P<0.05$ vs respective sham; ${ }^{\# P}<0.05$ vs respective sepsis; $+P<0.05$ vs respective $C T R L$.

Abbreviations: DIO, diet-induced obesity; CTRL, control; SEM, standard error of the mean.

\section{Adiponectin treatment attenuates circulating cell-endothelial cell interactions in cerebral microcirculation}

We first studied the leukocyte and platelet adhesion in cerebral microcirculation in DIO vs CTRL mice. As shown in Figure 1, leukocyte adhesion in the cerebral microcirculation of sepsis mice was significantly increased compared to that in sham mice in both DIO and CTRL groups. We also showed that the leukocyte adhesion in the DIO-sepsis mice was significantly increased compared to CTRL-sepsis mice. Adiponectin treatment significantly attenuated the leukocyte adhesion in the cerebral microcirculation of both the DIO and CTRL mice compared to the sepsis alone mice. Leukocyte adhesion in sepsis + adiponectin groups from DIO vs. CTRL was not significantly different from each other.

Figure 2 depicts platelet adhesion in the cerebral microcirculation in DIO vs CTRL mice. Similar to leukocyte adhesion shown in Figure 1, platelet adhesion in sepsis mice was significantly increased compared to sham counterparts in both DIO and CTRL groups; platelet adhesion in DIO mice was significantly increased compared to CTRL mice with sepsis. Similarly, the platelet adhesion in adiponectin-treated mice was significantly attenuated compared to sepsis alone in both the DIO and CTRL mice. Platelet adhesion in sepsis + adiponectin groups from DIO vs CTRL was not significantly different from each other.

\section{Adiponectin treatment attenuates inflammatory response in $\mathrm{FAF}$ macrophages}

We have shown previously that adiponectin deficiency exaggerates microvascular inflammatory response via increased adhesion molecule expression in the microcirculation. ${ }^{19}$ Furthermore, we have demonstrated (Figures 1 and 2) that the

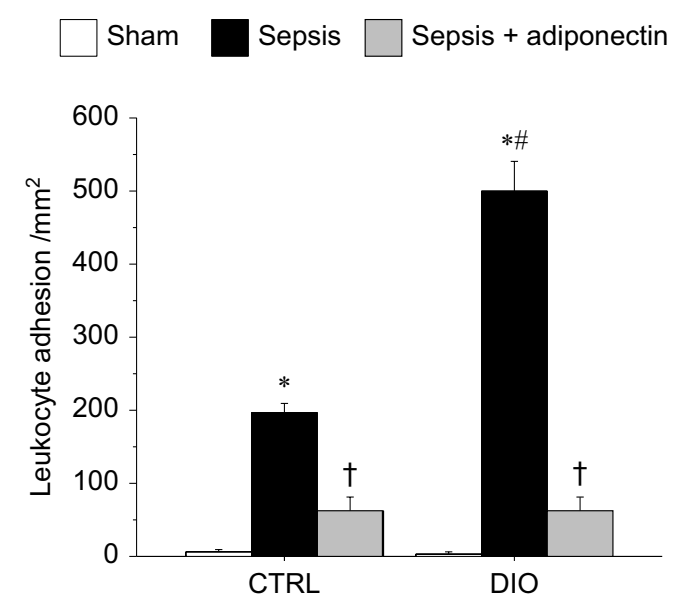

Figure I Adiponectin decreased leukocyte adhesion in cerebral microcirculation in CTRL and DIO mice with sepsis.

Notes: Sepsis-induced leukocyte adhesion in the cerebral microcirculation was significantly increased in DIO and CTRL mice compared to respective sham control. Moreover, the leukocyte adhesion in DIO-sepsis mice was significantly increased compared to the CTRL-sepsis mice. In both the CTRL and DIO mice, the leukocyte adhesion in adiponectin-treated mice was significantly decreased compared to their respective sepsis alone counterparts. Leukocyte adhesion in sepsis + adiponectin groups from CTRL vs DIO was not significantly different from one another. $* P<0.05$ vs respective sham; $\# P<0.05$ vs $C T R L$ sepsis; $\dagger P<0.05$ vs respective sepsis.

Abbreviations: CTRL, control diet; DIO, diet-induced obesity.

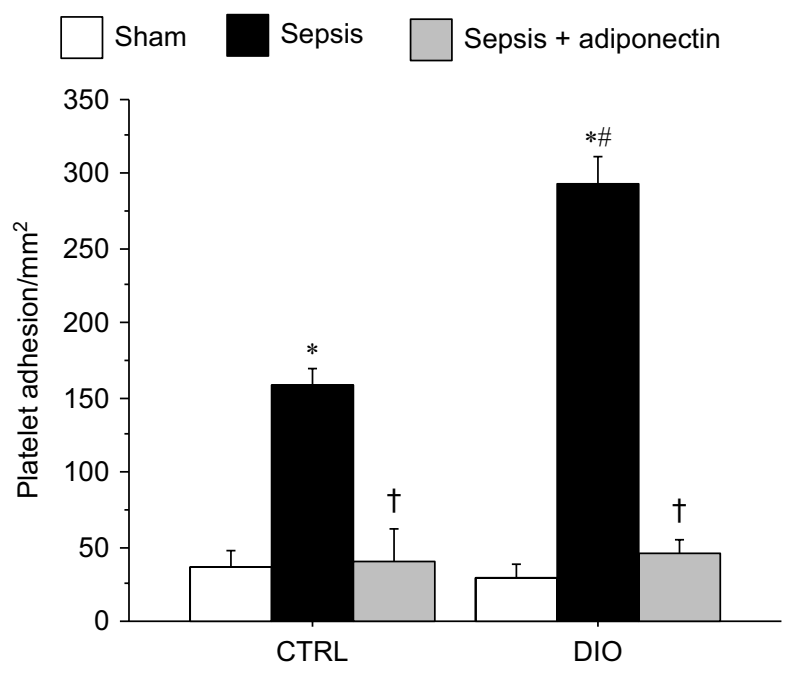

Figure 2 Adiponectin decreased platelet adhesion in cerebral microcirculation in CTRL and DIO mice with sepsis.

Notes: Sepsis-induced platelet adhesion in the cerebral microcirculation followed a similar pattern as in the leukocyte adhesion. There was a significant increase in platelet adhesion in both the DIO and CTRL mice compared to the respective sham control. Moreover, the platelet adhesion was significantly increased in DIO-sepsis mice compared to the CTRL-sepsis mice. In both the CTRL and DIO mice, the platelet adhesion in adiponectin-treated mice was significantly decreased compared to their respective sepsis alone counterparts. Platelet adhesion in the sepsis + adiponectin groups from CTRL vs DIO was not significantly different from one another. ${ }^{*} P<0.05$ vs respective sham; ${ }^{\sharp} P<0.05$ vs $C T R L$ Sepsis; ${ }^{\dagger} P<0.05$ vs respective sepsis.

Abbreviations: CTRL, control diet; DIO, diet-induced obesity.

high fat diet exaggerates microvascular inflammation and that adiponectin treatment attenuates circulating cell-endothelial cell interactions in the cerebral microcirculation. In the present study, we elucidated the effect of adiponectin treatment on immune cells with FFA feeding. Specifically, we studied 
the effect of adiponectin on BMDM cells with and without FFA feeding vs control (BSA). As shown in Figure 3, we demonstrated that in BMDM cells, FFA treatment significantly increased the TNF- $\alpha$ mRNA expression in response to LPS stimulation. Moreover, we also showed that adiponectin treatment attenuated TNF- $\alpha$ mRNA expression in response to LPS stimulation in both the BSA (control)- and FFA-fed macrophages, although the TNF- $\alpha$ mRNA expression in the adiponectin-treated FFA group continued to be significantly higher than in the adiponectin-treated BSA group.

\section{Adiponectin treatment increases SIRTI mRNA expression in FFA-treated macrophages}

The literature suggests that obesity is sirtuin-deficient state..$^{24}$ Several lines of evidence suggest that the proinflammatory or phenotype of obesity is modulated via decreased SIRT1 expression. We have shown that FFA-fed RAW cells showed decreased SIRT1 protein expression (data not shown).

SIRT1 has been implicated in the anti-inflammatory effect of adiponectin treatment earlier, ${ }^{25,26}$ however, the effect of adiponectin treatment on FFA-fed macrophages is unknown. Here, we studied SIRT1 mRNA expression in FFA- vs BSA (control)-fed macrophages with adiponectin/control treatment prior to LPS stimulation. As shown in Figure 4, in FFAfed cells, there was a significant increase in SIRT1 mRNA expression compared to BSA with and without adiponectin treatment. Moreover, adiponectin treatment in BSA-fed

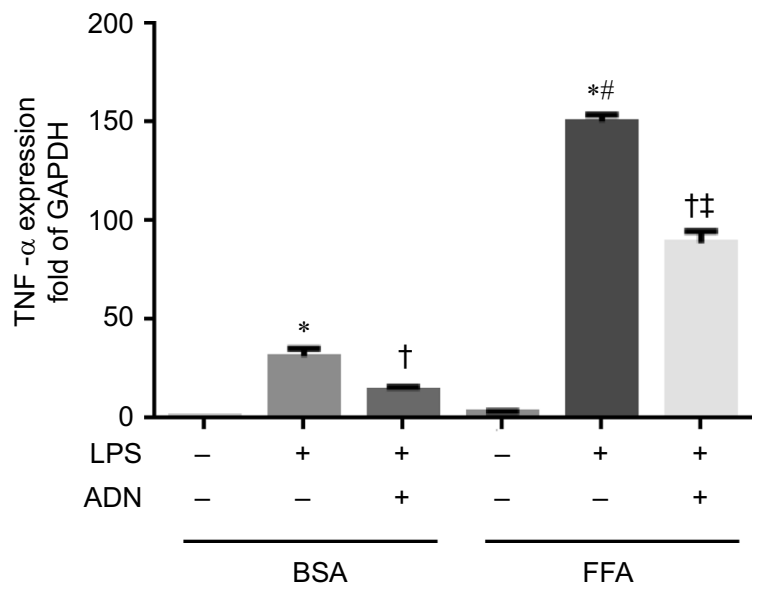

Figure 3 Adiponectin treatment attenuates inflammatory response in fatty acid-fed macrophages.

Notes: We demonstrate that in BMDM cells, FFA treatment significantly increased the TNF- $\alpha$ mRNA expression in response to LPS stimulation. Moreover, we also show that adiponectin treatment attenuated TNF- $\alpha$ mRNA expression in response to LPS stimulation in both the BSA control and FFA-fed macrophages. ${ }^{*} P<0.05$ vs respective BSA control; ${ }^{+P}<0.05$ vs respective LPS-stimulated group; ${ }^{\#} P<0.05$ vs BSA + LPS; ${ }^{P}<0.05$ vs $B S A+A D N$ group.

Abbreviations: BSA, bovine serum albumin; FFA, free fatty acid; LPS, lipopolysaccharide; TNF- $\alpha$, tumor necrosis factor-alpha; ADN, adiponectin.

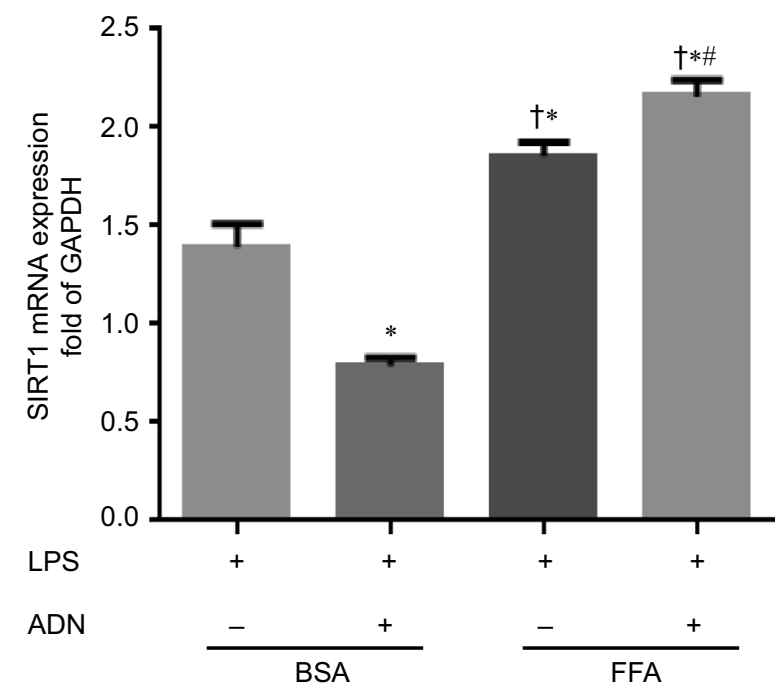

Figure 4 Adiponectin treatment and SIRTI mRNA expression.

Notes: There was a significant increase in the expression of SIRTI mRNA in FFA-fed macrophages vs BSA-fed macrophages. Moreover, adiponectin treatment showed decreased SIRTI mRNA expression in BSA-fed macrophages compared to FFA-fed macrophages; there was a significant increase in SIRTI mRNA expression with adiponectin treatment. ${ }^{*} P<0.05$ vs $B S A+L P S ;{ }^{+} P<0.05$ vs $B S A+L P S+A D N$; ${ }^{\#} P<0.05$ vs FFA + LPS.

Abbreviations: BSA, bovine serum albumin; FFA, free fatty acid; LPS, lipopolysaccharide; SIRTI, sirtuin-I; ADN, adiponectin.

macrophages showed decreased SIRT1 mRNA expression, while a significant increase in the expression was observed in FFA-fed macrophages. This suggests that SIRT1 plays an important role in the anti-inflammatory effect of adiponectin in macrophages.

\section{Adiponectin treatment attenuates inflammation via SIRTI-dependent and -independent pathways in obesity}

As shown in Figure 4, we observed that adiponectin increased SIRT1 mRNA expression in FFA-fed macrophages with LPS stimulation. In order to further elucidate the role of SIRT1 in adiponectin treatment, we isolated BMDM from myeloid-specific SIRT1KO mice, fed them with FFA vs BSA control, treated with or without adiponectin treatment prior to LPS stimulation, and studied TNF- $\alpha$ mRNA expression. As shown in Figure 5, in BSA-fed BMDM from WT mice, adiponectin treatment decreased TNF- $\alpha$ mRNA expression significantly, as expected, and the macrophages from SIRT1KO mice also showed decreased TNF- $\alpha$ mRNA expression with adiponectin, although the change did not reach statistical significance.

FFA-fed macrophages showed a significant increase in TNF- $\alpha$ mRNA expression in all groups compared to BSA-treated cells, indicating a proinflammatory phenotype in FFA-fed macrophages compared to BSA control. The macrophages from FFA-fed SIRT1KO mice showed a significant increase in TNF- $\alpha$ mRNA expression compared 


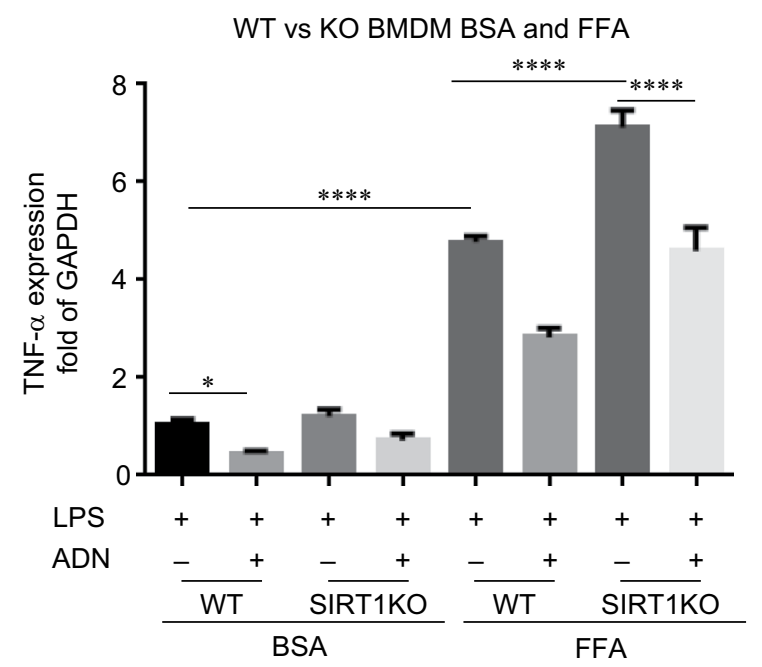

Figure 5 Bone marrow-derived macrophages with FFA and BSA feeding with and without adiponectin treatment.

Notes: BMDM isolated from WT and SIRTIKO mice showed increased TNF- $\alpha$ mRNA expression in response to LPS after BSA as well as FFA-feeding. Adiponectin treatment decreased TNF- $\alpha$ mRNA expression in SIRTIKO mice only in BSA-fed macrophage and not in FFA-fed macrophages. FFA feeding of BMDM showed that there was a significant increase in TNF- $\alpha$ mRNA in both WT and SIRTIKO groups vs respective WT groups. There was a significant increase in TNF- $\alpha$ mRNA in FFAfed SIRTIKO BMDM vs FFA-fed WT BMDM. Adiponectin treatment significantly decreased TNF- $\alpha$ mRNA expression in both WT and SIRTIKO mice with FFA feeding, although the TNF- $\alpha$ mRNA expression in ADN-treated SIRTIKO BMDM remained significantly higher vs WT BMDM. $* P<0.05$; **** $P<0.0001$.

Abbreviations: BSA, bovine serum albumin; BMDM, bone marrow-derived macrophages; FFA, free fatty acid; LPS, lipopolysaccharide; TNF- $\alpha$, tumor necrosis factor-alpha; SIRTIKO, SIRTI knockout; WT, wild type; SIRTI, sirtuin-I.

to those from BSA control in response to LPS. Adiponectin treatment in FFA-fed WT macrophages attenuated TNF- $\alpha$ mRNA expression compared to control as expected. Surprisingly, adiponectin treatment also decreased TNF- $\alpha$ mRNA expression in FFA-fed macrophages from SIRT1KO mice compared to control treatment. These data suggest that the adiponectin-induced attenuation of TNF- $\alpha$ mRNA expression in macrophages is via SIRT1-dependent as well as SIRT1independent pathways.

\section{Discussion}

Obesity increases morbidity and resource utilization in already expensive care of critically ill patients. ${ }^{2,27}$ Adiponectin, an adipokine secreted by differentiating adipocytes, is known to attenuate inflammatory response in early sepsis in the lean mice. Adiponectin deficiency is implicated in cardiovascular mortality in obese patients. ${ }^{12,28}$ Previously, we have shown that adiponectin deficiency exaggerates microvascular inflammatory response in early sepsis via modulation of the expression of endothelial adhesion molecules. ${ }^{19}$ Obesity is associated with adiponectin deficiency; we have previously shown the exaggeration of microvascular inflammation in obese mice with sepsis; however, the effect of adiponectin replacement on obese septic mice is not very well studied. To our knowledge, this is the first study to show the effect of adiponectin replacement on nutritionally obese mice with sepsis. In addition, we have shown, for the first time, the effect of adiponectin treatment on a "macrophage model of obesity", ie, BMDM treated with FFA.

Microvasculature sits at an interface between systemic circulation and tissue environment. Not surprisingly, leukocyte adhesion is a rate-determining factor in any inflammatory response, including that in sepsis. We have shown previously, using leptin deficient $\mathrm{ob} / \mathrm{ob}$ and leptin resistant $\mathrm{db} / \mathrm{db}$ mice, that obesity is associated with exaggerated microvascular leukocyte adhesion and adhesion molecule expression; moreover, adiponectin deficiency is associated with exaggerated microvascular inflammation via endothelial adhesion molecule expression. ${ }^{19}$ The literature suggests that adiponectin replacement attenuates inflammatory response in early sepsis in lean mice, ${ }^{29}$ but the effect of adiponectin replacement in sirtuin-deficient obese mice with sepsis is not well understood. In the present study, we showed that adiponectin replacement attenuates microvascular inflammation in nutritionally obese mice. Interestingly, we showed that along with the leukocyte adhesion, adiponectin treatment decreased platelet adhesion as well. The literature indicates that platelets do possess adiponectin receptors, and metabolic syndrome is associated with increased platelet aggregation via adiponectin deficiency; pretreatment of platelets with adiponectin attenuates platelet aggregation significantly. ${ }^{30,31}$ Our data are consistent with these findings.

Recent studies have indicated that LPS stimulation in mice is associated with decreased adiponectin receptors transiently;32 moreover, obesity is also associated with decreased expression of adiponectin receptors in adipose and skeletal muscle tissue. ${ }^{33,34}$ It is possible that obesity-related adiponectin deficiency could be related to the expression of adiponectin receptors. The role of adiponectin receptor expression in obese with sepsis needs further evaluation.

In this study, we also showed a novel cell model of obesity. We fed BMDM with FFA (stearic acid in this case) to further elucidate the mechanism of adiponectin treatment. Evidence suggests that obesity is a sirtuin-deficient state. ${ }^{24} \mathrm{We}$ have also demonstrated that the SIRT1-deficient ob/ob mice showed exaggeration of sepsis-induced inflammation via decreased SIRT1 protein expression; SIRT1 protein activation in ob/ob mice using resveratrol attenuated microvascular inflammation via increased SIRT1 protein expression. ${ }^{6}$ However, the effect of nutritional obesity on SIRT1 mRNA expression in immune cells is not very well understood. In this study, we showed that while the SIRT1 protein expression is known to be decreased in obesity ${ }^{24}$ there is a significant increase in SIRT1 mRNA expression in the FFA-treated BMDM, most likely as a positive feedback. 
We found that the SIRT1 mRNA expression in BSAtreated BMDM decreased in adiponectin-treated cells, while it increased in FFA-treated cells. This was a surprising finding. We have shown previously that SIRT1 is regulated by posttranslational modification and stabilization of SIRT1 protein during the late/hypoinflammatory phase of sepsis. ${ }^{35}$ It is highly likely that adiponectin helps stabilize the SIRT1 protein; however, the pharmacokinetics of the effect of adiponectin on SIRT1 protein stabilization and posttranslational modification is different in BSA- vs FFA-treated cells. This needs to be further elucidated with longer time course with specific focus on differential pharmacokinetics in BSA- vs FFA-treated macrophages.

The literature suggests that adiponectin attenuates inflammatory response in immune cells via AMP kinase pathway. While the connection between adiponectin and AMPK is well established, ${ }^{36}$ that between adiponectin and SIRT1 in obesity is not very well characterized. Our data suggest that FFA feeding with and without LPS stimulation is associated with increased SIRT1 mRNA expression. Therefore, we hypothesized that adiponectin treatment in the FFA-fed macrophages attenuates inflammatory response via modulation of SIRT1 expression. We have studied the effect of sirtuins on NFKB acetylation and NFKB-regulated genes previously. ${ }^{6,37,38}$ However, our data suggest that macrophages from SIRT1KO mice also show attenuation of inflammatory response, although to a lesser degree compared to WT mice. This suggests the presence of a SIRT1-independent pathway. Further work is required to determine whether this alternative pathway is AMP kinase pathway, although this is beyond the scope of the present study. There are unanswered questions in this project. First, we studied only the early inflammatory changes with and without adiponectin in DIO and CTRL mice. We did not study either late sepsis or survival in these mice. The effect of adiponectin treatment on late sepsis and on survival in mice with and without obesity needs to be further delineated. Second, we studied only SIRT1 pathway and did not study AMP kinase pathway. However, the connection between adiponectin and AMP kinase is a very well established one. ${ }^{36}$ Thus, the AMP kinase and other pathways need to be studied in obese mice and FFA-fed macrophages. Third, while we studied the mRNA expression, the effects of adiponectin on SIRT1 protein expression in obese mice and FFA-fed macrophages also need to be studied.

\section{Conclusion}

We show, for the first time, that adiponectin replacement attenuates microvascular inflammation in nutritionally obese, septic mice. Mechanistically, we studied the effect of adiponectin treatment on FFA-fed mouse macrophages and showed that adiponectin attenuates inflammatory response in immune cells via SIRT1-dependent and SIRT1-independent pathways. Future studies need to focus on the effect of adiponectin on SIRT1-independent pathways responsible in FFA-fed immune cells and obese mice.

\section{Acknowledgments}

Myeloid SIRT1 knockout (SIRT1KO) mice were generous gift from Dr Hang Shi (Georgia State University). The work was supported by NIH grants to Vidula Vachharajani (No R01GM099807) and Charles E McCall (Nos R01AI065791, R01AI079144).

\section{Disclosure}

The authors report no conflicts of interest in this work.

\section{References}

1. Gaieski DF, Edwards JM, Kallan MJ, Carr BG. Benchmarking the incidence and mortality of severe sepsis in the United States. Crit Care Med. 2013;41(5):1167-1174.

2. Prescott HC, Chang VW, O’Brien JM, Jr, Langa KM, Iwashyna TJ. Obesity and 1-year outcomes in older Americans with severe sepsis. Crit Care Med. 2014;42(8):1766-1774.

3. Aird WC. The role of the endothelium in severe sepsis and multiple organ dysfunction syndrome. Blood. 2003;101(10):3765-3777.

4. Jung U, Norman KE, Scharffetter-Kochanek K, Beaudet AL, Ley K. Transit time of leukocytes rolling through venules controls cytokine-induced inflammatory cell recruitment in vivo. J Clin Invest. 1998;102(8):1526-1533.

5. Vachharajani V, Russell JM, Scott KL, et al. Obesity exacerbates sepsisinduced inflammation and microvascular dysfunction in mouse brain. Microcirculation. 2005;12(2):183-194.

6. Wang X, Buechler NL, Yoza BK, McCall CE, Vachharajani VT. Resveratrol attenuates microvascular inflammation in sepsis via SIRT1-Induced modulation of adhesion molecules in ob/ob mice. Obesity (Silver Spring). 2015;23(6):z1209-1217.

7. Finkelstein EA, Khavjou OA, Thompson H, et al. Obesity and severe obesity forecasts through 2030. Am J Prev Med. 2012;42(6):563-570.

8. Wang HE, Griffin R, Judd S, Shapiro NI, Safford MM. Obesity and risk of sepsis: a population-based cohort study. Obesity (Silver Spring). 2013;21(12):E762-E769.

9. Eber MR, Laxminarayan R, Perencevich EN, Malani A. Clinical and economic outcomes attributable to health care-associated sepsis and pneumonia. Arch Internl Med. 2010;170(4):347-353.

10. Vincent JL, Marshall JC, Namendys-Silva SA, et al. Assessment of the worldwide burden of critical illness: the intensive care over nations (ICON) audit. Lancet Respir Med. 2014;2(5):380-386.

11. Vachharajani V, Granger DN. Adipose tissue: a motor for the inflammation associated with obesity. IUBMB Life. 2009;61(4):424-430.

12. Matsuda M, Shimomura I. Roles of oxidative stress, adiponectin, and nuclear hormone receptors in obesity-associated insulin resistance and cardiovascular risk. Horm Mol Biol Clin Investig. 2014;19(2): 75-88.

13. Shibata R, Ouchi N, Murohara T. Adiponectin and cardiovascular disease. Circ J. 2009;73(4):608-614.

14. de Boer MP, Meijer RI, Richter EA, et al. Globular adiponectin controls insulin-mediated vasoreactivity in muscle through AMPK $\alpha 2$. Vascul Pharmacol. 2015;78:24-35.

15. Ehsan M, Singh KK, Lovren F, et al. Adiponectin limits monocytic microparticle-induced endothelial activation by modulation of the AMPK, Akt and NFאB signaling pathways. Atherosclerosis. 2015;245:1-11. 
16. Famulla S, Schlich R, Sell H, Eckel J. Differentiation of human adipocytes at physiological oxygen levels results in increased adiponectin secretion and isoproterenol-stimulated lipolysis. Adipocyte. 2012;1(3):132-181.

17. Chan KH, Lam KS, Cheng OY, et al. Adiponectin is protective against oxidative stress induced cytotoxicity in amyloid-beta neurotoxicity. PLoS One. 2012;7(12):e52354.

18. Sakai S, Iizuka N, Fujiwara M, et al. Mild obesity reduces survival and adiponectin sensitivity in endotoxemic rats. J Surg Res. 2013;185(1): 353-363.

19. Vachharajani V, Cunningham C, Yoza B, Carson J, Jr, Vachharajani TJ, McCall C. Adiponectin deficiency exaggerates sepsis-induced microvascular dysfunction in the mouse brain. Obesity (Silver Spring). 2012; 20(3):498-504.

20. Vachharajani V, Vital S, Russell J, Scott LK, Granger DN. Glucocorticoids inhibit the cerebral microvascular dysfunction associated with sepsis in obese mice. Microcirculation. 2006;13(6):477-487.

21. Yang Z, Wang X, HeY, et al. The full capacity of AICAR to reduce obesityinduced inflammation and insulin resistance requires myeloid SIRT1. PLoS One. 2012;7(11):e49935.

22. Tailor A, Granger DN. Hypercholesterolemia promotes P-selectindependent platelet-endothelial cell adhesion in postcapillary venules. Arterioscler Thromb Vasc Biol. 2003;23(4):675-680.

23. Weischenfeldt J, Porse B. Bone marrow-derived macrophages (BMM): isolation and applications. CSH Protoc. 2008;2008:pdb prot5080.

24. Poulsen MM, Jorgensen JO, Jessen N, Richelsen B, Pedersen SB. Resveratrol in metabolic health: an overview of the current evidence and perspectives. Ann N Y Acad Sci. 2013;1290:74-82.

25. Costa Cdos S, Rohden F, Hammes TO, et al. Resveratrol upregulated SIRT1, FOXO1, and adiponectin and downregulated PPARgamma1-3 mRNA expression in human visceral adipocytes. Obes Surg. 2011;21(3):356-361.

26. Jiang Z, Zhou J, Zhou D, Zhu Z, Sun L, Nanji AA. The adiponectinSIRT1-AMPK pathway in alcoholic fatty liver disease in the rat. Alcohol Clin Exp Res. 2015;39(3):424-433.
27. Akinnusi ME, Pineda LA, El Solh AA. Effect of obesity on intensive care morbidity and mortality: a meta-analysis. Crit Care Med. 2008; 36(1):151-158.

28. Lindberg S, Jensen JS, Bjerre M, et al. Adiponectin, type 2 diabetes and cardiovascular risk. Eur J Prev Cardiol. 2015;22(3):276-283.

29. Teoh H, Quan A, Bang KW, et al. Adiponectin deficiency promotes endothelial activation and profoundly exacerbates sepsis-related mortality. Am J Physiol Endocrinol Metab. 2008;295(3):E658-E664.

30. Restituto P, Colina I, Varo JJ, Varo N. Adiponectin diminishes platelet aggregation and SCD40L release. Potential role in the metabolic syndrome. Am J Physiol Endocrinol Metab. 2010;298(5):E1072-E1077.

31. Kato H, Kashiwagi H, Shiraga M, et al. Adiponectin acts as an endogenous antithrombotic factor. Arterioscler Thromb Vasc Biol. 2006; 26(1):224-230.

32. Hall A, Leuwer M, Trayhurn P, Welters ID. Lipopolysaccharide induces a downregulation of adiponectin receptors in-vitro and in-vivo. PeerJ. 2015;3:e1428

33. Yamauchi T, Kadowaki T. Adiponectin receptor as a key player in healthy longevity and obesity-related diseases. Cell Metab. 2013;17(2):185-196.

34. Tsuchida A, Yamauchi T, Ito Y, et al. Insulin/Foxo1 pathway regulates expression levels of adiponectin receptors and adiponectin sensitivity. J Biol Chem. 2004;279(29):30817-30822.

35. Liu TF, Yoza BK, El Gazzar M, Vachharajani VT, McCall CE. NAD+dependent SIRT1 deacetylase participates in epigenetic reprogramming during endotoxin tolerance. J Biol Chem. 2011;286(11):9856-9864.

36. Sun Y, Li J, Xiao N, et al. Pharmacological activation of AMPK ameliorates perivascular adipose/endothelial dysfunction in a manner interdependent on AMPK and SIRT1. Pharmacol Res. 2014;89: 19-28.

37. Vachharajani VT, Liu T, Brown CM, et al. SIRT1 inhibition during the hypoinflammatory phenotype of sepsis enhances immunity and improves outcome. J Leukoc Biol. 2014;96(5):785-796.

38. Wang X, Buechler NL, Martin A, et al. Sirtuin-2 regulates sepsis inflammation in ob/ob mice. PLoS One. 2016;11(8):e0160431.
Journal of Inflammation Research

\section{Publish your work in this journal}

The Journal of Inflammation Research is an international, peer-reviewed open access journal that welcomes laboratory and clinical findings on the molecular basis, cell biology and pharmacology of inflammation including original research, reviews, symposium reports, hypothesis formation and commentaries on: acute/chronic inflammation; mediators of

\section{Dovepress}

inflammation; cellular processes; molecular mechanisms; pharmacology and novel anti-inflammatory drugs; clinical conditions involving inflammation. The manuscript management system is completely online and includes a very quick and fair peer-review system. Visit http://www.dove press.com/testimonials.php to read real quotes from published authors. 